\title{
DESARROLLO PSICOMOTOR EN UN GRUPO DE ESTUDIANTES DE 4 AÑOS DE EDUCACIÓN INICIAL DE LA RED 06-CALLAO
}

\author{
Hector Guillermo Aguinaga Espinoza* \\ Universidad San Ignacio de Loyola \\ dear_agus@hotmail.com
}

Fecha de recepción: agosto de 2014 Fecha de aceptación: octubre de 2014

ReSUmen: La presente investigación tuvo como propósito conocer el nivel de desarrollo psicomotor en un grupo los estudiantes de 4 años de las Instituciones de Educación Inicial de la Red 06 Callao. Se trata de un estudio descriptivo simple, con una muestra de 80 estudiantes del género masculino y femenino. Se utilizó el Test de Desarrollo Psicomotor (TEPSI) de las autoras Haeussler y Marchant (1994) adaptado por Aguinaga y Aguinaga (2011), que evalúa el nivel de desarrollo psicomotor general y en las dimensiones de motricidad, coordinación y lenguaje. Los resultados mostraron que los estudiantes se ubican en el nivel de normalidad en cuanto a su desarrollo psicomotor general y en cada una de sus dimensiones.

Palabras Clave: desarrollo psicomotor, lenguaje, coordinación, motricidad

* Hector Guillermo Aguinaga Espinoza es magíster en Educación con mención en Psicopedagogía Infantil por la Universidad San Ignacio de Loyola. Es bachiller y licenciado de Educación Primaria con mención en la Especialidad de Retardo Mental por la Universidad Nacional Mayor de San marcos. Asimismo, es titulado en Educación Primaria con mención en la Especialidad de Retardo Mental por el Instituto Superior Pedagógico Público de Educación Especial «María Madre». 


\title{
DEVELOPMENT PSYCHOMOTOR IN A GROUP OF STUDENTS OF 4 YEARS OLD OF INITIAL EDUCATION FROM THE RED 06-CALLAO
}

\begin{abstract}
Aвstract: This simple descriptive investigation aims to describe the psychomotor level of development in a group of 4 year-old students from Institutions of initial education of the Red 06 Callao. The sample were 80 students male and female. The Test of Psychomotor Development (TEPSI) of the authors Haeussler and Marchant (1994) adapted by Aguinaga and Aguinaga (2011), assessing the overall level of psychomotor development and dimensions of motor, coordination and language, was used. The results showed that most of students rated an average level in their general psychomotor in each of its dimensions.
\end{abstract}

KEYwORDs: psychomotor of development, motor, coordination, language.

\section{INTRODUCCIÓN}

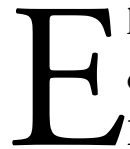

1 presente trabajo de investigación nace de la inquietud por saber cómo evoluciona el desarrollo psicomotor en los estudiantes de la Región Callao. Según refiere el Ministerio de Educación (MINEDU, 2011), un adecuado progreso de este permite que los acompañantes del niño (profesionales docentes, no docentes, padres de familia y comunidad) lo guíen en «el manejo eficiente de su cuerpo, en la construcción de sus pensamientos, el equilibrio físico, coordinaciones y la seguridad en sí mismo; con una autoestima que le permita enfrentar con eficacia y madurez las dificultades que se le presenten en el contexto en el que se desenvuelve» (MINEDU 2011: 7). 
DESARROLLO PSICOMOTOR EN UN GRUPO DE ESTUDIANTES DE 4 AÑOS DE EDUCACIÓN INICIAL DE LA RED 06-CALLAO

Teniendo como referencia lo citado anteriormente por el MINEDU, es necesario saber cómo está evolucionando el desarrollo psicomotor en los estudiantes. El motivo principal es que este es un aspecto de gran importancia para la obtención de futuros aprendizajes que le permitan enfrentar la vida. Es por ello que el presente estudio se planteó con el fin de conocer el desarrollo psicomotor en los estudiantes de 4 años de las Instituciones de Educación Inicial de la Red 06 Callao, en sus tres áreas básicas: (a) motricidad, (b) lenguaje y (c) coordinación.

Para conocer el nivel de desarrollo psicomotor, se estimóla utilización de un instrumento que evalúe las áreas de la variable antes mencionada; en este caso, se empleó el Test de Desarrollo Psicomotor (TEPSI), de las autoras chilenas Haeussler y Marchant (1994). El mencionado instrumento posee 52 ítems, divididos en tres áreas básicas: (a) motricidad, (b) lenguaje y (c) coordinación (Ver Tabla 1). Estos permiten ubicar a los estudiantes en tres niveles o categorías de desarrollo: (a) normalidad, (b) riesgo y (c) retraso, y conocer el estado actual del desarrollo psicomotor de los estudiantes en su totalidad como en sus áreas.

Además, para sustentar el estudio se ha recabado información sobre el desarrollo psicomotor. Con ello, se ha detallado desde la definición, evolución, las áreas que lo conforman y otros componentes relacionados al desarrollo psicomotor. Asimismo, se mencionan los antecedentes de estudios, tanto nacionales como internacionales, los cuales han servido de sustento para la comparación y efectividad del estudio.

Todo ello se ha realizado con el fin de organizar un marco teórico que pueda definir conceptos y teorías que sirvan de base para dos aspectos del estudio. Estos son los siguientes: (a) la comprensión y (b) discusión necesaria de la variable. 


\section{1 Problema de investigación}

El presente estudio plantea interrogantes. Estas proponen el problema general y los problemas específicos. A continuación se mencionan dichas interrogantes:

- Problema general: ¿Cuál es el nivel de desarrollo psicomotor en un grupo de estudiantes de 4 años de Educación Inicial de la red 06-Callao?

- Problemas específicos:

1) ¿Cuál es el nivel de desarrollo psicomotor en el área coordinación en un grupo de estudiantes de 4 años de Educación Inicial de la red 06-Callao?

2) ¿Cuál es el nivel de desarrollo psicomotor en el área lenguaje en un grupo de estudiantes de 4 años de Educación Inicial de la red 06-Callao?

3) ¿Cuál es el nivel de desarrollo psicomotor en el área motricidad en un grupo de estudiantes de 4 años de Educación Inicial de la red 06-Callao?

Teniendo en cuenta el Proyecto Educativo Nacional al 2021 (PEN) en el que se señala que tiene como uno de sus objetivos «asegurar el desarrollo óptimo de la infancia a través de la acción intersectorial concertada del Estado en cada región» (Consejo Nacional de Educación 2006: 16), el estudio se justifica porque los datos obtenidos aportan a la sociedad científica cifras y resultados que pueden servir de punto de partida para futuros estudios de investigación donde se pretenda estudiar la variable, compararla 
u observar si los resultados variaron con el pasar del tiempo. Además, se justifica en lo social y educativo, ya que hasta el momento se ha tomado como parámetro el desarrollo cognitivo desde el inicio de la escolaridad de los estudiantes, sin considerar el desarrollo psicomotor con respecto a sus estadios según investigaciones de Piaget (1936) y otros autores entre ellos Doussoulin (2003), Guralnick (1997) y Vigotsky (1934). Por último, el estudio posibilitará que la sociedad reconozca cuán importante resulta el desarrollo psicomotor y cómo este ayuda en el desarrollo de las habilidades de los estudiantes. Asimismo, contribuirá a que los docentes de inicial sean menos presionados por los padres, los cuales exigen el desarrollo de habilidades académicas, no teniendo en cuenta la etapa de desarrollo por la que atraviesan sus hijos. Este fenómeno acarrea que los docentes no puedan hacer el mejor uso de estas etapas como herramienta en el aprendizaje y formación de los estudiantes.

Finalmente, el estudio tiene como objetivo general describir el nivel de desarrollo psicomotor en el grupo de estudiantes antes mencionado, y tres objetivos específicos. Estos últimos son los que siguen: (a) describir el nivel de desarrollo psicomotor en el área coordinación en un grupo de estudiantes de 4 años de educación inicial de la red 06-Callao; (b) describir el nivel de desarrollo psicomotor en el área lenguaje en un grupo de estudiantes de 4 años de educación inicial de la red 06-Callao, y (c) describir el nivel de desarrollo psicomotor en el área motricidad en un grupo de estudiantes de 4 años de educación inicial de la red 06-Callao.

\section{El desarrollo psicomotor}

Se trata de la progresiva adquisición y organización de habilidades biológicas, psicológicas y sociales en el niño; además de ser la manifestación externa de 
la maduración del sistema nervioso central, la cual se traduce en cambios secuenciales, progresivos e irreversibles del individuo en crecimiento (Arteaga y cols. 2001; Moore 1996). Es así que el desarrollo psicomotor es un constante acomodo y reacomodo de lo que se aprende, alimentándose de experiencias previas para que la próxima vez que se realice una misma actividad se haga mejor. Además, es un proceso coordinado donde hay otros procesos que se desarrollan en el sistema nervioso central y quedan grabados, los cuales ayudan a la resolución de actividades de similares características en un futuro. Por ello, el progreso de este es el reflejo de la evolución del sistema nervioso central del individuo.

Por otro lado, el desarrollo psicomotor es esencialmente la educación del movimiento, o la educación por medio del movimiento, que procura una mejor utilización de las capacidades psíquicas que incluyen las intelectuales, otorgándole gran importancia a las experiencias sensoriomotoras y perceptivomotoras en el desarrollo de las competencias del aprendizaje. Tal como refirió Da Fonseca (2000), el desarrollo psicomotor puede ser modificado y ayudado mediante una educación que se centre en actividades que hagan experimentar al individuo situaciones donde tenga que resolver problemas, tales como intercalar pasos, definir movimientos estereotipados, etc. En tal sentido, el desarrollo del sistema nervioso central y el desarrollo psicomotor pueden ser ayudados externamente para lograr una mejor evolución a través del movimiento y así brindar mayores opciones de desarrollo de las habilidades psíquicas e intelectuales, asegurando los futuros aprendizajes.

Al saber lo anterior y con aporte teórico de Doussoulin (2003), se puede mencionar que el desarrollo psicomotor «es un proceso multidimensional de continuo cambio, en donde el niño comienza a 
dominar niveles cada vez más complejos en sus funciones» (2003: 70). Este incluye cambios en los siguientes planos: (a) físico o motor (su capacidad para dominar movimientos); (b) intelectual (su capacidad para pensar y razonar); (c) emocional (su capacidad para sentir); (d) social (su capacidad para relacionarse con los demás), y (e) sensorial (su capacidad para recibir los diferentes estímulos del medio). Son estas áreas del desarrollo del niño fundamentales para su maduración y crecimiento.

\section{Áreas de desarrollo psicomotor}

\section{a) Motricidad}

Se entiende por motricidad al movimiento considerado desde un punto de visto anatomo-fisiológico, en el cual se encuentran evidenciadas dos leyes que rigen a todas las especies, incluyendo a la humana: (a) la ley céfalo caudal y (b) la ley próximo distal. La primera refiere que el desarrollo se produce de arriba (cabeza) hacia abajo (pies), y la segunda que el desarrollo se produce del centro del cuerpo hacia la periferia (Cf. Vial 1972 y Portelland 1987, citados por Zavala 1991).

De acuerdo a Panéz (1989), el desarrollo motor depende de la maduración de las estructuras neuronales, óseas, musculares y cambios de las proporciones corporales. Además, el aprendizaje, cuando se ajusta a la maduración del individuo, es también un factor de importancia en el desarrollo de la motricidad. Asimismo, el investigador sostuvo que el desarrollo del control del cuerpo guarda relación con el desarrollo de las áreas motoras cerebrales, particularmente de los lóbulos frontales, que tienen la función de controlar los movimientos y se desarrollan durante los primeros años de la infancia. Es así que el cerebelo, que es el encargado de controlar el equilibrio, se desarrolla rápidamente durante los primeros años; 
particularmente en la segunda mitad del primer año y los primeros meses del segundo, momento en que el niño va a presentar capacidad para andar.

Por otro lado, la motricidad se divide en desarrollo motor grueso y desarrollo motor fino (Jiménez 1982). El primero comprende el control de partes gruesas del cuerpo para realizar diferentes movimientos. El control motor grueso es un hito en el desarrollo de un bebé, el cual puede refinar los movimientos descontrolados, aleatorios e involuntarios a medida que su sistema neurológico madura. El segundo abarca todas aquellas actividades en la que el niño necesita de una precisión y un elevado nivel de coordinación. El desarrollo o habilidad motora fina, de acuerdo con Maza y Arce (1991), comprende desde la prensión refleja a la destreza manipulativa y desde la fijación ocular al dominio visual completo. Ambas están interrelacionadas entre sí a través de la iniciativa ideomotriz, hasta el logro de una adecuada coordinación visomotriz. Asimismo, los autores también hicieron referencia a «componentes básicos de la motricidad fina»: el componente manual y el componente visual.

Cabe destacar que al desarrollar los dos componentes antes mencionados, surge la prensión. Esta es un reflejo que el niño posee desde el nacimiento y que al desarrollarse se considera como la prensión verdadera, ya que esta necesita una coordinación óculo-manual y un dominio de los músculos óculo-motores; además de la voluntariedad del acto motor. La prensión presenta una etapa importante en el desarrollo del niño que le permitirá la apropiación del mundo exterior por manipulación y descubrimiento (Cf. Maza y Arce 1991).

b) Coordinación

Para Lorenzo (2006), la coordinación motriz es el conjunto de capacidades que organizan y regulan de forma precisa todos los procesos parciales de 
un acto motor en función de un objetivo motor preestablecido. Dicha organización se ha de enfocar como un ajuste entre todas las fuerzas producidas, tanto internas como externas, considerando todos los grados de libertad del aparato motor y los cambios existentes del contexto.

Además, la coordinación es el factor primario de la localización espacial y de las respuestas direccionales precisas, en que las percepciones de los sentidos juegan un papel importante en el desarrollo, ya que van a ser la base de la coordinación. Esto es debido a que primero existe una capacidad temprana de formar esquemas mentales, productos de la experiencia con el medio, a través de las cuales se forma patrones para la coordinación y de regulación propioceptiva-vestibular.

Cabe destacar que los movimientos dependen de los estímulos que provienen de los centros vestibulares centrales. Es así que a medida que el individuo se va desarrollando aparecen movimientos más precisos y más localizados. Las coordinaciones más precisas, en general, se establecen a los seis años de edad cronológica, aunque es razonable encontrar aún en ese periodo algunos movimientos agregados, que son aislados y se llaman sincinecias (alteración en el desarrollo psicomotor).

c) Lenguaje

Es una de las funciones psicológicas que más roles desempeña en el desarrollo psíquico del ser humano. Permite comunicar y expresar emociones. De igual forma, interviene en los procesos cognitivos: (a) pensamiento, (b) memoria, (c) razonamiento, (d) solución de problemas y (e) planeamiento (Vygotsky 1934).

Asimismo, el lenguaje es la unidad de las funciones comunicativas y representativas del entorno, que tiene un desarrollo específico con raíces propias en la comunicación prelinguística y que no depende necesariamente 
del desarrollo cognitivo, sino de la interacción con su medio. Así, el lenguaje es una función que se adquiere a través de la relación del individuo con su entorno y respaldado biológicamente por las estructuras necesarias que posee para crear signos de comunicación verbal. En ese sentido, el proceso evolutivo lleva a los seres humanos a manejar instrumentos (símbolos), como el lenguaje, para adaptarse a su entorno. Por tanto, la conducta humana está organizada y controlada, tanto por intenciones reales (del propio individuo) como por intenciones atribuidas por otros (provenientes de la interacción con los demás) (Cf. Sapir 1921 citado por Vygotsky 1934).

Por otro lado, Vigotsky (1934) rescató la participación del niño en actividades culturales con la guía de compañeros más capaces; porque estas le permiten al niño interiorizar los instrumentos necesarios para pensar y acercarse a la resolución de algún problema de un modo más maduro, que pondría en práctica si actuara por sí solo. En otras palabras, lo que el niño interioriza es lo que previamente ha realizado en el contexto social. De esta manera, la creación cultural canaliza las destrezas de cada generación, y con ello el desarrollo individual está mediado por la interacción con otras personas más hábiles (padres, maestros o compañeros del colegio) en el uso de los instrumentos culturales como lo es el lenguaje.

\section{Alteraciones del desarrollo psicomotor}

Las alteraciones del desarrollo psicomotor se dan por anomalías en el proceso de evolución. Estas anomalías retrasan el desarrollo normal, debido a que impiden que se sigan las secuencias dadas en el proceso de desarrollo. Los niños como todos los individuos siguen una secuencia determinada en su desarrollo con la adquisición de habilidades determinadas por patrones claros y definidos en hitos básicos, fáciles de medir y que permiten 
identificar el progreso secuencial. Por lo tanto, «en casos donde se evidencia la falta de la adquisición de habilidades, anomalías y retraso en el desarrollo, se puede determinar que es causada por una alteración en el desarrollo psicomotor, especialmente cuando se presenta directamente relacionado con la maduración del sistema nervioso central» (Doussoulin 2003: 72).

Las alteraciones del desarrollo psicomotor determinan las anomalías en dos factores. Por un lado, deja sentada la configuración biológica o herencia, la cual es el factor que determina el potencial del niño de existir la presencia de un daño establecido en el sistema nervioso central o condiciones biológicas; estos en un futuro impedirán el desarrollo normal de este. Por otro lado, estarían los factores de riesgo ambiental, los cuales influyen si un niño es criado en un ambiente pobre y carente de estímulos; así, las interacciones neuronales establecidas resultarán ser menos adaptativas al momento de responder a un medioambiente en constante cambio. Además, existe un conjunto de variables asociadas al nivel socioeconómico, que podrían ir en desmedro del desarrollo del niño; por ejemplo, constitución de la familia, escolaridad de los padres, situación de la vivienda, trabajo del sostenedor del hogar y desconocimiento de una estimulación adecuada (Doussoulin 2003: 73).

Cabe mencionar que la alteración en el desarrollo psicomotor produce déficit en la parte motora al influir en el desarrollo de tareas o actividades que requieren precisión en relación con el esquema corporal (espacio y tiempo), el cual requiere de mayor tiempo para ser asimilado y utilizado en la vida cotidiana. Por otra parte, estas alteraciones en el desarrollo psicomotor influyen en el mal funcionamiento del aspecto sensorial, al no percibir el espacio y tiempo, ya que no logra interpretar las sensaciones físicas siguientes: (a) tamaño, (b) distancia, (c) forma y (d) 
sucesos. Por último, la alteración producida en el lenguaje, es decir, cuando el niño tarda en hablar e inclusive deja de hablar por motivos neurológicos o por el contexto social, puede ocasionar que el niño hable mal, tenga un retraso simple del lenguaje e incluso un trastorno específico del lenguaje o tartamudez. Estos son problemas del lenguaje causados por un entorno que afectan aspectos en el autoestima del niño (MINEDU 2011: 11).

\section{Psicomotricidad en la educación inicial}

Según refiere el MINEDU en el Diseño Curricular Nacional de Educación básica regular (2009) entre los 0 y 2 años, los niños presentan un notorio crecimiento físico y desarrollo de las habilidades motoras tanto gruesas como finas, que van de la mano con los cambios que se dan en las áreas cognitiva, afectivas y simbólicas. Por esta razón, los niños requieren de espacios adecuados para favorecer este desarrollo motor, lo cual en el caso de las zonas urbanas está limitado a diferencia de las zonas rurales y amazónicas. Estas últimas ofrecen condiciones y posibilitan todos sus movimientos de manera natural y con mayor libertad, debido a que el desarrollo de las habilidades motoras - desde levantar la cabeza y el tórax en los primeros meses, hasta voltearse, sentarse y alcanzar objetos - lleva al incremento del contacto con el medio.

En otro punto, el MINEDU (2009) menciona que en los niños entre los 6 y 12 meses se desarrolla la habilidad motora para desplazarse gateando y caminando, así como para manipular, jalar y abrir los objetos con mayor coordinación y precisión. Entre los 12 y 18 meses realiza las actividades siguientes: (a) un mayor desplazamiento en el entorno: ya camina, trepa, sube peldaños, patea pelotas, entre otras actividades; (b) sigue la trayectoria de los objetos con la mirada, los tira y se desplaza para 
DESARROLLO PSICOMOTOR EN UN GRUPO DE ESTUDIANTES DE 4 AÑOS DE EDUCACIÓN INICIAL DE LA RED 06-CALLAO

encontrarlos, y (c) manipula materiales diversos con una intención, como introducirlos en recipientes, encajarlos en moldes, etc. Entre los 18 y los 24 meses, el desplazamiento se orienta por el reconocimiento de las posiciones en el espacio: dentro, fuera, arriba, abajo, encima, al lado, abierto, cerrado, delante y detrás.

Por otro lado, el MINEDU (2009) menciona que entre los 2 y 4 años el desarrollo de las habilidades motoras le permite al niño mayor balance del cuerpo para lanzar y patear pelotas, impulsarse para brincar un peldaño, pedalear, saltar sobre dos pies y sobre un pie, entre otros. Estas experiencias contribuyen al desarrollo cognitivo a través de la percepción, la imitación y los esquemas mentales. También, repite los movimientos aprendidos con el propósito de provocar un efecto deseado. Anticipa lo que va a suceder como consecuencia de su acción, y la respuesta la va coordinando en sus esquemas mentales.

Por último, el MINEDU (2009) incide en que a partir de los 3 años, el niño realiza muchas preguntas sobre las cosas, por lo que se denomina la «edad de los por qué». Memoriza intencionalmente la información que obtiene como respuesta a sus preguntas y a su exploración del medio. Entre los 4 y 5 años, el desarrollo motor le permite al niño mayor actividad, como galopar, atrapar y rebotar una pelota, arrastrarse en el piso, mantener el equilibrio en estructuras tipo vigas, nadar, cazar, pescar, cabalgar, etc. Asimismo, el desarrollo neuromuscular le permite dibujar formas, copiar círculos y cuadrados, ensartar cuentas u otros objetos, usar tijeras para cortar, apilar bloques, vestirse solo y abotonarse (motricidad fina).

Es así que el MINEDU (2009), en el nivel inicial, plantea, teniendo en cuenta el aspecto psicomotor, competencias acorde a la edad de los niños. Propone que a los 3 años los niños deben ser preparados para realizar diversos 
movimientos mostrando lo siguiente: (a) control postural, (b) equilibrio, (c) seguridad física, (d) ritmo, (e) control tónico, (f) coordinación motriz, (g) respiración y $(\mathrm{h})$ tono muscular. A los 4 años, deben ser preparados para realizar diferentes actividades donde se observen las siguientes cualidades del movimiento: (a) control tónico, (b) control del equilibrio, (c) coordinación motriz, (d) respiración, (e) tono muscular y (f) velocidad. Finalmente, a los 5 años, los niños deberán estar dispuestos a realizar diferentes actividades en las que se evidencie lo que a continuación se menciona: (a) control tónico, (b) control del equilibrio, (c) coordinación motriz, (d) respiración, (e) tono muscular, (f) fuerza, (g) flexibilidad y (h) velocidad. Además, aprecia los movimientos de sus pares y los imita para lograr mayor dominio corporal.

En tal sentido, los estudiantes están en el momento clave de su vida, en la cual se puede detectar a tiempo si se encuentran en una situación de riesgo en cuanto a su desarrollo y poder realizar la intervención oportuna y adecuada. Al respecto, Alcover (2010) sostuvo que «los autores insisten en la necesidad de detectar estas dificultades ya en la etapa preescolar, cuando se instauran importantes bases cognitivas como la conducta que junto al funcionamiento motor, sentarán los pilares para el posterior éxito social y académico» (2010: 37). Asimismo, menciona que «entre los 3 y 4 años se empieza a poder medir la inteligencia, así como el desarrollo de conceptos, habilidades pre académicas, indicadores tempranos de funciones ejecutivas y capacidades de integración viso motoras. También pueden diferenciarse mejor las habilidades verbales de las no verbales» (2010:36).

\section{MÉTODO}

El tipo de investigación utilizado en este trabajo es la llamada descriptiva. Los procedimientos de esta fueron explicados por Danhke (citado por 
Bisquerra, 2004) «como aquellos que buscan especificar las propiedades, las características y los perfiles importantes de personas, grupos, comunidades o cualquier otro fenómeno que se someta a un análisis» (Bisquerra 2004: 114). Así, el estudio pretendió identificar las características de la variable desarrollo psicomotor en los estudiantes de 4 años de las Instituciones de Educación Inicial de la Red 06 Callao.

Además, el diseño que se empleó fue el descriptivo simple. Este "constituye una opción cuantitativa que trata de realizar descripciones precisas y muy cuidadosas respecto a fenómenos educativos» (Bisquerra 2004: 197). Es por ello que en este caso se recolectó datos, sin manipular la variable, sobre el desarrollo psicomotor de los estudiantes de 4 años para describirla.

Formalización:

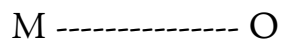

Donde:

$\mathrm{M}=$ Estudiantes de 4 años.

$\mathrm{O}=$ Desarrollo psicomotor.

Por otro lado, la variable de investigación por estudiar es el desarrollo psicomotor. Este se define de manera conceptual y operacional. A continuación, se explicarán estas ideas.

- Definición conceptual: El desarrollo psicomotor es la evolución de las capacidades para realizar una serie de movimientos corporales y acciones, así como la representación mental y consciente de los mismos. En este desarrollo hay unos componentes madurativos, relacionados con la maduración cerebral, y unos componentes relacionales que tienen que ver con el hecho de que a través de su 
movimiento y sus acciones el sujeto entre en contacto con personas y objetos con los que se relaciona de manera constructiva (Cf. Monge s.f.).

- Definición operacional: Se trata del puntaje obtenido por los estudiantes en las dimensiones de coordinación, lenguaje y motricidad en el test de Desarrollo Psicomotor (Cf. Haeussler y Marchant, 2002). 
DESARROLLO PSICOMOTOR EN UN GRUPO DE ESTUDIANTES DE 4 AÑOS DE EDUCACIÓN INICIAL DE LA RED 06-CALLAO

Tabla 1

Dimensiones e indicadores del Test de Desarrollo Psicomotor Dimensiones Indicadores

Traslada agua de un vaso hacia otro sin derramar.

Construye un puente dejando una abertura entre dos cubos.

Construye una torre con más de 8 cubos sin apoyo.

Desabotona los dos botones de un estuche.

Abotono los dos botones de un estuche.

Enhebra una aguja sin apoyo.

Coordinación

Desata la amarra.

Dibuja una línea recta, vertical y horizontal.

Dibuja un círculo con un solo movimiento.

Dibuja dos líneas rectas que se intercepten en el medio formando una cruz.

Dibuja un triángulo con ángulos bien formados.

Dibuja un cuadrado con sus ángulos bien rectos.

Dibuja una figura humana con más de 3 partes.

Ordena objetos por tamaños encajándolas en un tablero. 
Señala en una lámina la figura grande y pequeña.

Señala en una lámina la agrupación con más y menos objetos.

Nombra animales que observa en una lámina.

Nombra objetos que observa en una lámina.

Señala en una lámina la línea larga y corta.

Menciona la acción que realiza las personas de una lámina.

Menciona la utilidad de los objetos que se le muestra en una lámina.

Diferencia la bolsa pesada y la bolsa liviana.

Dice su nombre y apellido.

Indica verbalmente su sexo.

Menciona los nombres de sus padres.

Menciona las acciones que realiza ante las situaciones planteaLenguaje das.

Coloca objetos en diferentes posiciones.

Completa las frases con analogías opuestas.

Nombra el color del cuadrado que se le indica.

Muestra la figura del color que se le indica.

Dice el nombre de la figura geométrica que se le indica.

Señala la figura geométrica que se le indica.

Nombra acciones y sustantivos que se presentan en una lámina.

Verbaliza absurdos presentados en una lámina.

Menciona en plural una agrupación de objetos.

Identifica en láminas los hechos que ocurren antes y después de una escena.

Enuncia definiciones de objetos por alguna característica.

Nombra características de objetos mostrados. 
DESARROLLO PSICOMOTOR EN UN GRUPO DE ESTUDIANTES DE 4 AÑOS DE EDUCACIÓN INICIAL DE LA RED 06-CALLAO

Motricidad Da saltos seguidos con los pies juntos.

Camina llevando un vaso lleno con agua sin derramar.

Lanza con una mano la pelota hacia un punto que se le indica.

Permanece parado en un pie sin apoyo.

Camina en punta de pie.

Salta sobre un objeto con los pies juntos.

Salta con un pie con o sin avance y sin apoyo.

Coge la pelota con las dos manos.

Camina hacia adelante en línea recta con o sin apoyo tocando talón y punta.

Camina atrás en línea recta con o sin apoyo tocando talón y punta.

Nota: Variable controlada: Edad: 4 años

\subsection{Participantes}

La población estuvo conformada por 720 estudiantes de las aulas de 4 años de edad de las instituciones educativas A, B, C, y D. Estas pertenecían a la red 06-Callao. Dicha zona abarca la jurisdicción de la Ciudad Satélite de Santa Rosa-Callao.

La muestra es la no probabilística de tipo intencional, debido a que la elección de los sujetos se realizó de acuerdo con la edad, con los primeros 20 estudiantes y con la lista de cada aula. Por lo tanto, no dependió de la probabilidad, sino de las características de la investigación o de quién hace la muestra (Cf. Hernández, Fernández y Baptista 2006). Esta estuvo conformada por 80 estudiantes de 4 años de edad, distribuidos en 44 estudiantes del género masculino y 36 estudiantes del género femenino (ver Tabla 2). La muestra se obtuvo seleccionando un aula del turno mañana de 
cada una de las cuatro instituciones educativas del nivel Inicial pertenecientes a la Red 06-Callao.

Tabla 2

Distribución de la muestra por género

\begin{tabular}{lcc}
\hline Género & F & $\%$ \\
\hline Masculino & 44 & 55 \\
Femenino & 36 & 45 \\
Total & 80 & 100 \\
\hline
\end{tabular}

$\mathrm{N}=80$

\subsection{Descripción de la prueba}

El instrumento utilizado en la presente investigación fue el Test de Desarrollo Psicomotor (TEPSI), de las autoras Haeussler y Marchant (1985), docentes de la Pontificia Universidad de Chile. El TEPSI evalúa el desarrollo psicomotor de niños y niñas entre los 2 y 5 años de edad en tres áreas: (a) coordinación con 16 ítems, (b) lenguaje con 24 ítems y (c) motricidad con 12 ítems, por medio de la técnica de la observación de la conducta del niño o niña frente a las situaciones propuestas por el investigador (ver Apéndice 1). La aplicación requiere que sea individual, y el total se obtiene cuantificando los resultados para luego ser interpretados en una tabla de normas. Finalmente, según los resultados, se puntualiza y designa el nivel de desarrollo psicomotor de acuerdo con las siguientes categorías: (a) retraso, (b) riesgo y (c) normalidad. 
propuestas sí son consistentes con la variable que se pretende medir, según la opinión de los jueces consultados.

La confiabilidad del estudio se obtuvo, en la escala original de Haeussler y Marchant (1985), a través del análisis de dificultad e índice de discriminación de los ítems con el coeficiente de correlación biserial puntual (r.b.p.=.28), y el análisis del grado de dificultad del test y de los subtests. Asimismo, la consistencia interna del instrumento fue analizada a través del índice Kuder Richardson 20 y se obtuvieron los resultados que siguen: (a) .94 para el total del test, (b) .89 para el subtest de coordinación, (c) .94 para el subtest de lenguaje y (d) .82 para el subtest de motricidad. También, se realizó la concordancia interexaminador, donde se obtuvo los siguientes coeficiente $r$ de Pearson: (a) de .98 para el total del test, (b) .93 para el subtest de coordinación, (c) .97 para el subtest de lenguaje y (d) $.95 \mathrm{el}$ subtest para motricidad.

En el presente estudio se obtuvo la confiabilidad del instrumento utilizando el Alfa de Cronbach. Se obtuvo un valor ritc superior a .20 para todos los ítems, y un Alfa de Cronbach general de .918. En cuanto a las dimensiones, se consiguieron los resultados que siguen: (a) para el área de coordinación se obtuvo .763, (b) para el área de lenguaje se obtuvo .874 y (c) para el área de motricidad se obtuvo .836 .

\subsection{Procedimientos de recolección de datos}

Los procedimientos que se llevaron a cabo para la recolección de datos fueron tres. En primer lugar, se presentó un oficio remitido por la Dirección Regional de Educación del Callao (DREC) a las instituciones educativas, documentos con los que las directoras se informaron y dieron el visto bueno para la aplicación del instrumento de la investigación. En segundo lugar, se 
realizó las coordinaciones con las directoras de las instituciones de educación inicial de la red 06-Callao, para la entrevista con las docentes de las aulas por evaluar, quienes accedieron a brindar todas las facilidades del caso, $y$

depuraron las nóminas para conformar la muestra por investigar y el grupo para la prueba piloto. Finalmente, se evaluó a cada estudiante perteneciente a la muestra con el instrumento de investigación. La aplicación del TEPSI se ejecutó en cuatro semanas, abarcando un tiempo aproximado por estudiante de 30 a 40 minutos. Además, las docentes brindaron un ambiente adecuado para aplicar las diferentes pruebas del test. La aplicación del test a los estudiantes se realizó de manera personal por el autor de la investigación.

\subsection{Procedimientos de análisis de datos}

Los procedimientos que se llevaron a cabo para el análisis de datos fueron dos. Primero, se realizó la construcción de la base de datos en el programa SPSS v19 y se ingresaron los resultados obtenidos por ítem, áreas y puntajes totales. Segundo, se procesó y analizó estadísticamente los datos con SPSS v19, utilizando estadísticos descriptivos de frecuencia, media y desviación estándar.

\section{RESULTADOS}

\subsection{Resultados descriptivos}

En la Tabla 4, se aprecia las media y desviación estándar según áreas. Así, en el de coordinación presenta una media de 3.73 y una desviación estándar de .44; en el área de lenguaje, una media de 2.97 y una desviación estándar de .22 , y en el área de motricidad, una media de 3.23 y una desviación 
estándar de .42. Además, la media total del desarrollo psicomotor tiene un valor de 3.53 y una desviación estándar de .50. Dichos datos evidencian que la mayor desviación de los datos se muestra en el puntaje total del desarrollo psicomotor, y la menor dispersión se da en el área del lenguaje, la cual, a su vez, es la que presenta menor puntaje en la media.

Tabla 4

Media y desviación estándar según áreas y total del desarrollo psicomotor

\begin{tabular}{lcl}
\hline Medida & M & DE \\
\hline Coordinación & 3,73 & .44 \\
Lenguaje & 2,97 & .22 \\
Motricidad & 3,23 & .42 \\
Total & 3,53 & .50 \\
\hline
\end{tabular}

Por otro lado, en la Tabla 5, se observa que en el área de coordinación 80 estudiantes se ubican en la categoría de normalidad (100\%) y ninguno en riesgo o retraso. En el área de lenguaje, 77 estudiantes se ubican en la categoría de normalidad (96.3\%), 3 en la categoría de riesgo (3.8\%) y ningún estudiante en la categoría de retraso. En el área de motricidad, 80 estudiantes se ubican en la categoría de normalidad (100\%) y ninguno en riesgo o retraso. Finalmente, se observa que en el total del desarrollo psicomotor, 80 estudiantes se ubican en la categoría de normalidad (100\%) y ninguno en riesgo o retraso. 
DESARROLLO PSICOMOTOR EN UN GRUPO DE ESTUDIANTES DE 4 AÑOS DE EDUCACIÓN INICIAL DE LA RED 06-CALLAO

Tabla 5

Frecuencias y porcentajes en las áreas y categorias y en la variable del desarrollo psicomotor

\begin{tabular}{lcccc}
\hline \multicolumn{4}{c}{ Áreas del desarrollo psicomotor } \\
\hline & Coordinación & Lenguaje & Motricidad & Total \\
\hline Normali- & $80(100 \%)$ & $77(96.3 \%)$ & $80(100 \%)$ & $80(100 \%)$ \\
dad & & & & \\
Riesgo & $0(0 \%)$ & $3(3.8 \%)$ & $0(0 \%)$ & $0(0 \%)$ \\
Retraso & $0(0 \%)$ & $0(0 \%)$ & $0(0 \%)$ & $0(0 \%)$ \\
\hline
\end{tabular}

\subsection{Resultados complementarios}

En la Tabla 6, se observa que tanto en el área de coordinación como de motricidad 44 estudiantes hombres se encuentran dentro de la categoría de normalidad (100\%). En el área de lenguaje, 41 estudiantes hombres se encuentran dentro de la categoría de normalidad (93.2\%), mientras 3 estudiantes en la categoría de riesgo (6.8\%).

Tabla 6

Frecuencia y porcentaje según género masculino en las áreas y categorías del desarrollo psicomotor

\begin{tabular}{lccc}
\hline \multicolumn{3}{c}{ Masculino } \\
\hline & Coordinación & Lenguaje & Motricidad \\
\hline $\begin{array}{l}\text { Normali- } \\
\text { dad }\end{array}$ & $44(100 \%)$ & $41(93.2 \%)$ & $44(100 \%)$ \\
Riesgo & $0(0 \%)$ & $3(6.8 \%)$ & $0(0 \%)$ \\
Retraso & $0(0 \%)$ & $0(0 \%)$ & $0(0 \%)$ \\
$n=44$ & & &
\end{tabular}


En la Tabla 7, se observa que en las tres áreas del desarrollo psicomotor, 36 estudiantes mujeres se encuentran dentro de la categoría de normalidad (100\%). En la Tabla 8, se observa que 44 estudiantes hombres se encuentran dentro de la categoría de normalidad (100\%) y 36 estudiantes mujeres se ubican en la categoría de normalidad (100\%).

\section{Tabla 7}

Frecuencia y porcentaje según género femenino en las áreas y categorias del desarrollo psicomotor

\begin{tabular}{lccc}
\hline \multicolumn{3}{c}{ Femenino } \\
\hline Normali- & Coordinación & Lenguaje & Motricidad \\
dad & $36(100 \%)$ & $36(100 \%)$ & $36(100 \%)$ \\
Riesgo & $0(0 \%)$ & $0(0 \%)$ & $0(0 \%)$ \\
Retraso & $0(0 \%)$ & $0(0 \%)$ & $0(0 \%)$ \\
\hline$n=36$ & & &
\end{tabular}

\section{Tabla 8}

Frecuencia y porcentaje por género en las categorias del desarrollo psicomotor

\begin{tabular}{lcc}
\hline \multicolumn{2}{c}{ Género } \\
\hline Normalidad & Masculino & Femenino \\
Riesgo & $44(100 \%)$ & $36(100 \%)$ \\
Retraso & $0(0 \%)$ & $0(0 \%)$ \\
\hline
\end{tabular}

$n=80$ 
En la Tabla 9, se observa que los puntajes obtenidos en el área de coordinación por el género masculino presenta una media de 14.54 de los 16 ítems propuestos en el TEPSI y una desviación estándar de 1.45; mientras que el género femenino presenta en esta área una media de $14.41 \mathrm{y}$ una desviación estándar de 1.42. En el área de lenguaje, el género masculino presenta una media de 20.84 de los 24 ítems propuestos en el TEPSI y una desviación estándar de 2.23; mientras que género femenino presenta una media con un valor de 20.52 y una desviación estándar de 2.07. Por último, en el área de motricidad, el género masculino presenta una media de 10.38 de los 12 ítems propuestos en el TEPSI y una desviación estándar de 10.38.; mientras que el género femenino presenta una media de $10.05 \mathrm{y}$ una desviación estándar de 1.65.

Tabla 9

Media y desviación estándar en las áreas del desarrollo psicomotor según género

\begin{tabular}{|c|c|c|c|c|}
\hline \multicolumn{5}{|c|}{ Género } \\
\hline & \multicolumn{2}{|c|}{ Masculino } & \multicolumn{2}{|c|}{ Femenino } \\
\hline & M & $\mathrm{DE}$ & M & $\mathrm{DE}$ \\
\hline $\begin{array}{l}\text { Coordi- } \\
\text { nación }\end{array}$ & 14.54 & 1.45 & 14.41 & 1.42 \\
\hline Lenguaje & 20.84 & 2.23 & 20.52 & 2.07 \\
\hline Motricidad & 10.38 & 10.38 & 10.05 & 1.65 \\
\hline$n=80$ & & & & \\
\hline
\end{tabular}




\section{DISCUSIÓN}

La presente investigación se enmarca dentro del ámbito educativo, específicamente en lo que plantea el Proyecto Educativo Nacional al 2021PEN, el cual entre sus objetivos propone asegurar el desarrollo óptimo de la infancia a través de la acción intersectoral concertada del estado en cada región. Para los fines que persigue la investigación, se realizó la adaptación del Test de Desarrollo Psicomotor (TEPSI), cuyas autoras son las chilenas Haeussler y Marchant (1985). La confiabilidad del instrumento fue determinada a través del coeficiente del Alfa de Cronbach, cuyo resultado fue .299; de esta manera queda demostrado que el instrumento con 52 ítems es válido y altamente confiable.

Es cierto que se logró medir el nivel de desarrollo psicomotor de los estudiantes de cuatro años de Instituciones de educación inicial de la red 06-Callao, a través del TEPSI en sus tres áreas básicas: (a) motricidad, (b) lenguaje y (c) coordinación, llegando a arrojar como resultado total que 80 estudiantes de la muestra que representan el 100\% están en un nivel de normalidad, ya que los puntajes obtenidos fueron iguales y superiores a 40 ítems bien respondidos. Esto muestra que los estudiantes no han resuelto bien cada uno de los ítems del test; además, se dio el caso de que muchos estuvieron dentro del rango de aprobación para el nivel de normal y respondieron erróneamente algunos ítems, pero estos no fueron significativos para afectar en su evaluación. Sabiendo esto, se puede concluir que los estudiantes de 4 años de la red 06-Callao están logrando un desarrollo psicomotor normal. Asimismo, según los resultados obtenidos por Soler, Rivera, Figueroa, Sánchez y Sánchez (2007), se puede inferir que hay factores externos como los que siguen: (a) la estimulación sensorial, (b) la comunicación con sus compañeros, (c) padres y otras personas de su entorno, 
y (d) experiencias en situaciones motoras y de resolución de problemas, los cuales intervienen favorablemente en el desarrollo psicomotor y que, de acuerdo al MINEDU (2011), permitirán al niño ser un individuo cada vez más dueño de sí, y capaz de insertarse adecuadamente en el mundo que lo rodea. Incluso, se pueden asociar los resultados a procesos en el niño, ya que el buen funcionamiento del desarrollo psicomotor se dan por dos aspectos: (a) el genético, que engloba la carga genética y le brinda la predisposición que tiene el cuerpo en relación al desarrollo del sistema nervioso central, y (b) el aspecto ambiental, el cual comprende el aspecto externo y provee de estímulos que le brinda la experiencia, el contexto social, el contacto con agentes sociales sean estos positivos o negativos para su evolución normal en el desarrollo del sistema nervioso central, su procesamiento sensorial e integración sensorial y, por ende, su desarrollo psicomotor (Cf. JohnsonEcker y Parham 1999).

En el área de coordinación, los 80 estudiantes mostraron en un porcentaje de 100 que se encuentran ubicados en el nivel de normalidad. Esto demuestra, según Robles (2007), que cuando se estimula más el nivel de coordinación, este se potencia y mejora significativamente. También, siguiendo Lorenzo (2006), que la coordinación es la muestra externa de los ajustes dentro de la organización psíquica del individuo, la cual se está dando de buena manera, integrando tanto los factores internos como externos para la libertad en la ejecución de acciones coordinadas del aparato motor.

Por otro lado, 77 de los estudiantes en el área de lenguaje dieron un porcentaje de 96.3 en el nivel de normalidad y 3 estudiantes un porcentaje de 3.8 en el nivel de riesgo. Con lo que arroja esta información, se debería intervenir con un programa de estimulación y nivelación en esta área en los 3 estudiantes; esto debido a que, según el estudio realizado por Robles 
(2007), el lenguaje junto a la coordinación dan grandes resultados cuando se les estimula y potencian. Además, se debe tener en cuenta que al presentarse un 3.8 de porcentaje de la muestra en riesgo en el área, no quiere decir que presente problemas funcionales, sino que, como mencionó Vigostky (1934), «la guía de compañeros más capaces permite al niño interiorizar los instrumentos necesarios para pensar y acercarse a la resolución de algún problema que se presente» (1934: 24). Por otra parte, se puede asumir esto como un factor o característica del niño, ya que, según refirió Doussoulin (2003), cada infante posee su propia secuencia de desarrollo que está en directa relación con su maduración, por lo cual puede variar en características y calidad entre un niño y otro. En conclusión, los estudiantes con nivel de riesgo deben recuperar o desarrollar esas habilidades no presentadas en el momento del testeo más adelante y cuando deba darse según sus características propias como individuo. Esto se deduce teniendo en cuenta la información antes mencionada, la evaluación global que se hizo a los estudiantes y la situación de no encontrar estudiantes en el nivel de peligro. Finalmente, en el área de motricidad y en el total del desarrollo psicomotor, el $100 \%$ de los estudiantes se ubicaron en el nivel de normalidad. Es importante enfatizar en esta parte de la medición, ya que al comparar estos datos con los obtenidos en investigaciones, como en el caso de Moreyra y Prado (2010), en los cuales se evidenciaban hasta casos de retraso en el desarrollo, se debe asumir que los estudiantes de la red 06-Callao están logrando desarrollar un buen nivel psicomotor. Por ende, en el área de motricidad, la maduración normal de las estructuras neuronales, según Zavala (1991), también debe estar desarrollando normalmente la ley céfalo caudal y la ley próximo distal. 
La descripción de los niveles de desarrollo alcanzados por los estudiantes eran los esperados, y estos se pueden haber dado debido a diversos factores que intervienen: (a) la zona en la que viven, (b) la etapa biológica por la que pasan y (c) el nivel de Institución educativa donde cursan sus estudios preparativos para su vida educativa. También, cabe señalar que durante la evaluación y contacto con las docentes de educación inicial se observó que no conocen y dominan instrumentos de evaluación acorde a su nivel, llegando a fabricar ellas mismas sus pruebas. No obstante, la interrogante está en que si estas pruebas tomadas dan verdaderamente un resultado confiable como el TEPSI u otro instrumento de evaluación del desarrollo psicomotor en estudiantes de este nivel.

\section{CONCLUSIONES Y SUGERENCIAS}

El desarrollo psicomotor del grupo que participó en la investigación, a pesar de tener ítems errados en su evaluación, han logrado ubicarse en el nivel de normal. Es así que 80 estudiantes que representan el 100\% de la muestra evaluada están logrando un adecuado desarrollo psicomotor en los aspectos que siguen:

- El nivel de desarrollo de los estudiantes en el área coordinación es normal en 80 estudiantes, que representan el 100\% de la muestra de investigación.

- El nivel de desarrollo de los estudiantes en el área lenguaje es normal en 77 estudiantes, que representan el 96.3\% de la muestra, mientras que 3 estudiantes, que representan el 3.8\% de la muestra, se ubican en el nivel de riesgo en esta área de la investigación. 
- El nivel de desarrollo de los estudiantes en el área motricidad es normal en 80 estudiantes, que representan el 100\% de la muestra de investigación.

\subsection{Sugerencias}

Para próximas investigaciones en la Región Callao, se sugiere lo siguiente:

- Realizar estudios comparativos del desarrollo psicomotor de los estudiantes del nivel inicial pertenecientes a las redes del Callao.

- Realizar estudios de correlación entre el desarrollo psicomotor y el rendimiento escolar de estudiantes de zonas urbanas y marginales de la región Callao.

- Realizar estudios de correlación entre el desarrollo psicomotor y el grado de autoestima de estudiantes de estudiantes de zonas urbanas y marginales de la región Callao.

- Realizar estudios de correlación donde se analice el desarrollo psicomotor con las siguientes variables: (a) la edad, (b) tipo de vivienda, (c) constitución familiar y (d) lugar y (e) lengua de origen. 
DESARROLLO PSICOMOTOR EN UN GRUPO DE ESTUDIANTES DE 4 AÑOS DE EDUCACIÓN INICIAL DE LA RED 06-CALLAO

\section{Apéndice 1 \\ Instrumentos de investigación}

\section{Ficha técnica}

\section{Test de desarrollo psicomotor (TEPSI)}

Autoras

Año

Procedencia

Aplicación

Ámbito de aplicación

Adaptación

Año

Procedencia

Ámbito de aplicación

Finalidad

Materiales

Tipificación

Técnica de medición

Puntuación
: Isabel Haeussler P. y Teresa Marchant O.

: 1985 (1. ${ }^{a}$ edición)

: Santiago de Chile - Chile

: Individual

: Desde los 2 años, 0 meses, 0 días hasta los 5 años, 0 meses, 0 días.

: Elizabeth Aguinaga E. y Hector Aguinaga E.

: 2011

: Callao-Perú

: Desde los 4 años, 0 meses, 0 días hasta los 4 años 6 meses, 0 días.

: Evaluación del rendimiento psicomotor del niño, específicamente coordinación, lenguaje y motricidad gruesa

: - Una batería de prueba.

- Un manual de administración.

- Un protocolo u hoja de registro.

: Baremos de estudiantes escolares.

: Observación y registro de la conducta.

: $\quad \mathrm{PB}=$ Puntaje bruto (1 punto por acierto y 0 puntos por fracaso).

$\mathrm{T}=$ Puntaje $\mathrm{T}$ del total del test $\mathrm{y}$ por dimensión. 
HECTOR GUILLERMO AGUINAGA ESPINOZA

Subtest coordinación

16 pts.

Subtest lenguaje

24 pts.

Subtest motricidad

12 pts.

Puntuación Total máxima

52 pts. 
DESARROLLO PSICOMOTOR EN UN GRUPO DE ESTUDIANTES DE 4 AÑOS DE EDUCACIÓN INICIAL DE LA RED 06-CALLAO

\section{REFERENCIAS}

Alcover, Elena

$2010 \quad$ Seguimiento del desarrollo psicomotor de prematuros extremos mediante la Escala de Desarrollo Infantil de Kent (EDIK) cumplimentada por los padres y situaciones neuroevolutiva a los 2 y 5 años. Tesis de doctorado no publicada. Universidad de Barcelona, España. Disponible en <http://www.tesisenred.net/ bitstream $/$ handle/10803/2505/EAB_TESIS.pdf?sequence $=1>$. Consulta realizada en 30/01/2012.

Consejo Nacional de Educación

2006 La educación que queremos para el Perú-PEN-2021. Lima: CONADE.

Da FonseCA, Vítor

2000 Estudio y génesis de la psicomotricidad. Barcelona: Inde.

DANHKE, Gordon

1989 Investigación y comunicación. México, D.F.: Mc Gray Hill. 
Gobierno Regional Callao

$2000 \quad$ Plan de desarrollo de las capacidades humanas de la Región Callao 2005-2011. Disponible en <http://www.regioncallao. gob.pe/contenidos/contenidosGRC/filesContenido/PDCH\%20 Callao\%202005-2011.pdf>. Consulta realizada en 10/08/2012.

Haeussler, Isabel y Teresa Marchant

1985 Test de Desarrollo Psicomotor 2 a 5 años. Santiago de Chile: Universidad Pontificia De Chile $1^{\circ}$ edición.

1994 Test de Desarrollo Psicomotor 2 a 5 años. Santiago de Chile: Universidad Pontificia De Chile $10^{\circ}$ edición.

Hernández, Roberto, Fernández, Carlos y Pilar Baptista

2006 Metodología de la investigación. México, DF: Mc Graw Hill. $5^{\circ}$ edición.

JiMÉNEZ, Juan

1982 Neurofisiología psicológica fundamental. Barcelona: Científico médica.

Johnson-Ecker, C. y L. Parham

2000 "The Evaluation of Sensory Procedssing: A Validity Study Using Contrasting Groups». American Journal of Occupational Therapy, vol. 54, N. 5, Sep-Oct, pp. 494-503 
DESARROLLO PSICOMOTOR EN UN GRUPO DE ESTUDIANTES DE 4 AÑOS DE EDUCACIÓN INICIAL DE LA RED 06-CALLAO

LORENZO, Flaviano

2006 Coordinación motriz. Disponible en <http://www.efdeportes. com/efd93/coord.htm>. Consulta realizada en 23/01/2008.

Maza, Carlos y Carlos Arce

1991 Ordenar y clasificar. Madrid: Síntesis.

Ministerio de Educación

2011 Orientaciones para el desarrollo psicomotriz del niño con necesidades educativas especiales. Lima: Punto \& Grafía S.A.C.

2009 Diseño Curricular Nacional-Educación Básica Regular. Lima: Punto \& Grafía S.A.C.

Monge, Ma.

s.f. $\quad$ Desarrollo psicomotor como elemento fundamental en el desarrollo integral de niños y niñas en edades tempranas». Universidad de Costa Rica. Disponible en <http://www.edufi. ucr.ac.cr/pdf/ing/art2.pdf>.Consulta realizada en 24/07/2012.

MOORe, Rosario

1996 Evaluación del Desarrollo Psicomotor. Apuntes de Pediatría Pontificia Universidad Católica de Chile. Disponible en $<$ http://escuela.med.puc.cl/paginas/publicaciones/manualped/ EvalDessPs.html>. Consulta realizada en 15/06/2012. 
Moreyra, Nelly. y R. Prado

2010 Relación entre la contaminación ambiental por plomo y el crecimiento $y$ desarrollo de niños de 4-5 años en dos instituciones educativas iniciales en la región Callao. Tesis de licenciatura no publicada. Universidad Cayetano Heredia. Lima, Perú.

PANÉZ, Rosario

1989 Bajo el sol de la infancia. Lima: CONCYTEC.

Piaget, Jean

1936 El nacimiento de la inteligencia en el niño. Madrid: Crítica.

RoBLEs, Herbert

2008 «La Molina: La coordinación y motricidad asociada a la madurez mental en niños de 4 a 8 ańos». Avances en Psicología, revista de la Facultad de Psicología y Humanidades de la Universidad Femenina del Sagrado Corazón, vol. 16, N. 1, enero-diciembre, pp.139-154.

2007 Estudio del estado nutricional y el desarrollo psicomotriz en un grupo de niños de 3 y 4 años de una institución Educativa Inicial de Ate Vitarte. Tesis de maestría no publicada. Universidad Nacional de Educación Enrique Guzmán y Valle. Lima, Perú. 
DESARROLLO PSICOMOTOR EN UN GRUPO DE ESTUDIANTES DE 4 AÑOS DE EDUCACIÓN INICIAL DE LA RED 06-CALLAO

Soler, Karla; Rivera Iván; Figueroa Miriam; Sánchez Leonor y María SÁNCHEZ

2007 Relación entre las caracteristicas del ambiente psicosocial en el hogar y el desarrollo psicomotor en el niño menor a 36 meses de edad. Disponible en <http://www.medigraphic.com/pdfs/bmhim/ hi-2007/hi075c.pdf vista>. Consulta realizada en 27/01/2011.

UNESCO

2000 Dakar. Foro Mundial sobre la Educación: marco de acción de Dakar. Roma: Graphoprint.

VYGOTSKY, Lev

1934 Pensamiento y lenguaje. Buenos Aires: Nueva Visión.

ZaVAla, María

1991 Desnutrición y Desarrollo Psicomotor de Niños en Edad Pre-Escolar de Zonas Urbano-Marginales de Lima Metropolitana. Tesis de licenciatura no publicada. Universidad Ricardo Palma. Lima, Perú. 